\title{
Neutral-Pion Photoproduction on the Proton with the CLAS Detector
}

\author{
P. Heimberg \\ The George Washington University
}

for the CLAS Collaboration

\begin{abstract}
Absolute total and differential cross sections of the $\gamma N \rightarrow \pi N$ reaction channels may be used to determine partial wave amplitudes and photocouplings for the baryon resonances which decay predominantly into $N \pi$. The aim of the current analysis of the $\gamma p \rightarrow p \pi^{0}$ channel is to determine absolute differential and total cross sections to a precision of $5 \%$ (systematic) over an energy range of 0.4 to $1.8 \mathrm{GeV}$. In a single hour of running time, the CLAS collects several hundred thousand single- $\pi^{0}$ events, making this reaction ideal for studying systematic uncertainties in both the CLAS and the photon tagger. Identification of events coming from $\gamma p \rightarrow p \pi^{0}$ requires only the identification of a proton with correct missing mass. Nearly complete angular distributions can be obtained over most of the energy range, allowing the reliable determination of the total cross section. The evolution of the differential cross section is well reproduced by the SAID partial-wave analysis even up to about $1,7 \mathrm{GeV}$ where there is but modest existing experimental data from this channel to constrain the fit.
\end{abstract}

\section{SINGLE PION PHOTOPRODUCTION}

Below about $1 \mathrm{GeV}$ and at modest momentum transfer, the dynamics of strong interactions is reasonably well described in terms of (colorless) mesons and baryons. When probe wavelengths decrease to below the size of the nucleon, it becomes necessary to incorporate the more fundarnental degrees-of-freedom of quarks and gluons. Pion production can be exploited, both to examine the details of how certain baryon resonances are composed, and to determine when such processes become better described by perturbative QCD. Toward the former, a comprehensive and accurate database on single pion production on the nucleon $(\gamma N \rightarrow \pi N)$ would allow the precise determination of resonance width and photocouplings through the resonance region. Photocouplings are a sensitive testing ground for quarkbased calculations of nucleon structure $[1,2]$. One can also study this process without direct reference to intermediate resonances by looking for the onset of scaling derived from quark counting rules. In the region of high transverse momentum, QCD can make testable predictions for coherent production of pions on the proton as well as light nuclei $[3,4]$.

The current data set was obtained using tagged photons with energies between 0.4 and $3.1 \mathrm{GeV}$ on proton and deuteron targets, and 0.4 to $1.6 \mathrm{GeV}$ photons on ${ }^{3} \mathrm{He}$. This paper represents an analysis of the $\gamma p \rightarrow \pi^{0} p$ channel obtained from a run period covering 0.4 to $1.7 \mathrm{GeV}$.

\section{THE EXPERIMENT}

The data for this reaction were obtained using the Tagged-Photon Beam Facility [5] in conjunction with the CEBAF Large-Acceptance Spectrometer (CLAS) [6] in Hall-B at Jefferson Lab. 


\section{Tagged Photon Beam}

The photon beam is produced using a Bremsstrahlung radiator upstream of a dipole magnet which is backed by two planes ("E" and "T") of highly-segmented plastic scintillator detectors. This segmentation delivers a photon energy resolution of about $0.1 \%$ the energy of the incident electron beam.

The photon flux $\left(\sim 10^{7}\right.$ tagged $\left.\gamma / \mathrm{s}\right)$ is determined in a very simple way. Low-current runs are used to determine the tagging efficiency relative to a total-absorption photon counter (TAC) for each of the 61 T-counters (back plane) in the tagger. The tagging efficiency then connects the T-counter scalers with the photon flux during a high-current production run. This technique was fruitfully employed in a photofission experiment [7] to obtain the flux with an accuracy of $2 \%$ for each T-counter.

\section{The CLAS}

The CLAS is a toroidal magnetic spectrometer with charged-particle detectors subtending approximately $3 \pi$ steradians $\left(10<\theta_{l a b}<150^{\circ}\right)$. In the forward direction $\left(10<\theta_{\text {lab }}<45^{\circ}\right)$, there also exist electromagnetic calorimeters, employed in the present reaction for the detection of photons from $\pi^{0}$ decay.

A liquid hydrogen target ( $18 \mathrm{~cm}$ long) is located at the center of the CLAS. Surrounding the target is a set of thin plastic scintillators which provides a signal used to form a coincidence trigger with the tagger and provide a start for time-of-flight measurements.

Identification of events coming from $\gamma p \rightarrow p \pi^{0}$ is very straightforward, requiring only the identification of a proton and a missing mass equal to that of the $\pi^{0}$. One could also require one or two additional photons to be measured. And while this does eliminate a small $(<10 \%)$ background contamination, the acceptance becomes greatly reduced due to the forward location of the electromagnetic calorimeters. This is especially true at lower energies where a direct comparison with the world data set is desired. When photons are not required in the final state, nearly complete angular distributions can be obtained over the entire energy range, allowing the reliable extrapolation to the total cross section.

The detection volume is deternined using a GEANT-based Monte-Carlo simulation of the CLAS (GSIM [8]). Figure 1 shows the missing mass squared from the reaction $\gamma p \rightarrow p X$. The measured spectrum is shown in solid points while the histogram is the simulation for phase-space distributed $\gamma p \rightarrow p \pi^{0}$ events. The resolution is reproduced and shows the small level of background contamination from double pion production channels.

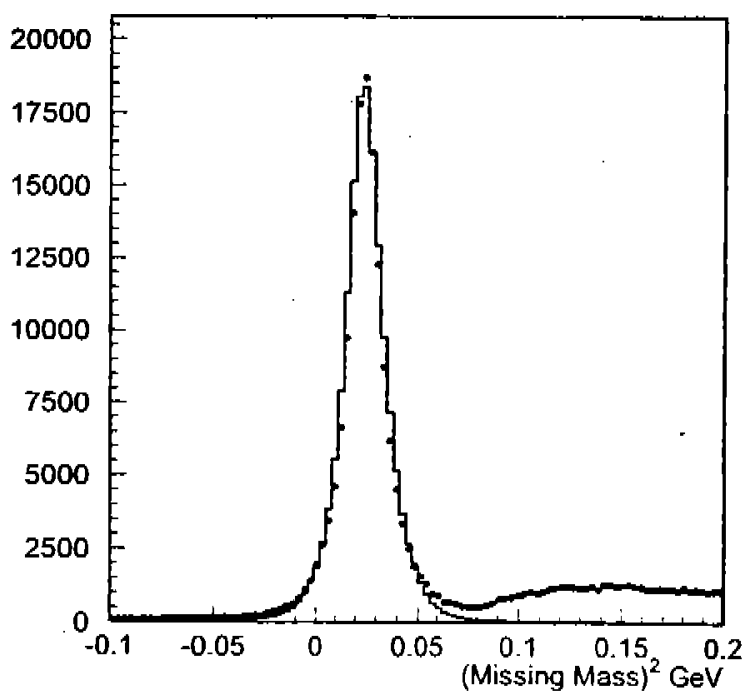

FIGURE 1. Missing mass squared with a single detected proton. Solid points are data from a single one-hour run while the histogram is a Monte-Carlo simulation of $\gamma p \rightarrow p \pi^{0}$ events. 


\section{PRELIMINARY RESULTS}

In extracting differential cross sections, cuts on missing mass, acceptance boundaries, and proton momentum were applied. The combined effect of such cuts is to remove about $10 \%$ of the yield and is accounted for in the simulation.

FIGURE 2. PRELIMINARY center-of-mass angular differential cross sections for the $\gamma p \rightarrow p \pi^{0}$ reaction. Only a few of the
available energy bins are shown here.

Figure 2 shows a sample of the differential cross sections obtained. The evolution of the differential cross section reproduces well that given by SAID [9] in the region where existing data provide significant constraints for the fit (up to about $1.2 \mathrm{GeV}$ ). Even up to the maximum energy of $1.7 \mathrm{GeV}$, the agreement is good, indicating that a sufficient number of resonant (and non-resonant) amplitudes are included in the phase shift analysis.

Exploiting the fact that the SAID fit does a reasonable job reproducing the shape of the angular distributions, the total cross section is obtained by scaling the SAID total cross section by the ratio of cross sections integrated over the acceptance, viz,

$$
\sigma_{t o t} \simeq \frac{\int_{C L A S} d \sigma^{E X P}}{\int_{C L A S} d \sigma^{S A I D}} * \sigma_{t o t}^{S A I D}
$$
The result of this procedure is shown in Figure 3 . The presence of the $P_{33}, D_{13}, F_{15}$, and $F_{37}$ baryon resonance groups
is evident.

In all cross section plots, the displayed error bars represent statistical uncertainty only. The systematic uncertainty is, at this point, estimated to be about $10 \%$ with the major contributors being uncertainties in proton efficiency and 


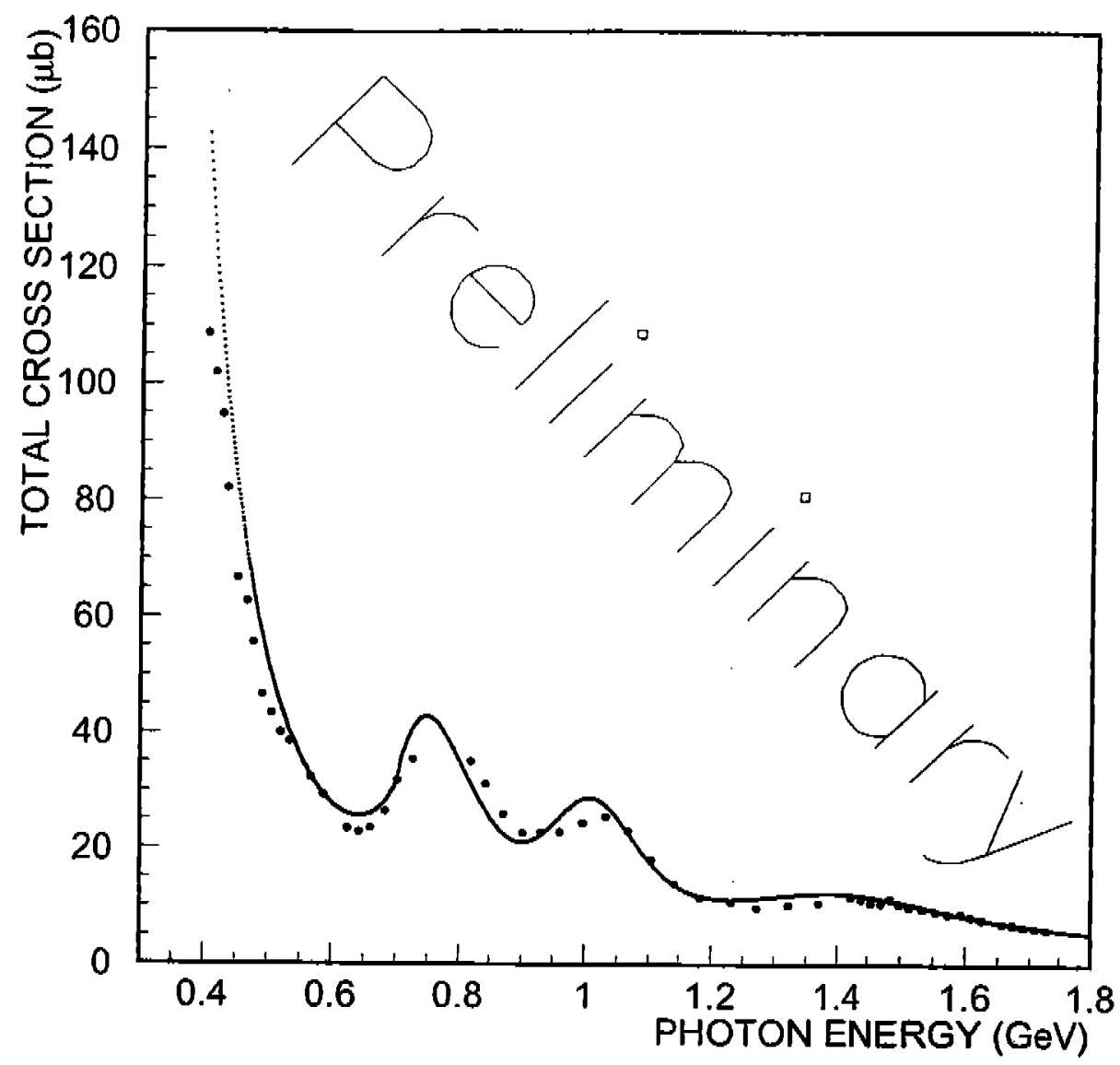

FIGURE 3. PRELIMINARY total cross sections for the $\gamma p \rightarrow p \pi^{0}$ reaction.

photon flux at about $7 \%$ and $4 \%$, respectively. Ultimately, the systematic uncertainties are expected to be about $6 \%$ in total.

\section{CONCLUDING REMARKS}

The data shown here represent approximately $1 \%$ of the data obtained on the proton and the analysis of these additional data (reaching up to $3.1 \mathrm{GeV}$ ) is well underway. Such statistical precision will permit very narow binning in both $E \gamma$ and $\theta_{\pi}^{*}\left(10 \mathrm{MeV}\right.$ and $5^{\circ}$, respectively). In concert with the $p \pi^{0}$ analysis, we are also extracting the $\pi^{+} n$ final state with equally promising initial results.

In addition, similar data sets have been acquired using both deuterium and ${ }^{3} \mathrm{He}$ targets, allowing a systematic study of coherent pion production over a broad kinematical range on several nuclei, from hard to soft processes. Furthermore, the quasifree pion- photoproduction channels $\left(\gamma d \rightarrow n p \pi^{0}, p p \pi^{-}\right)$will allow the extraction of the elementary amplitudes on the neutron.

Presently, the first experiment using the Hall-B linearly-polarized tagged photon beam and a proton target is underway. Photon asymmetries in the single pion channels will contribute complimentary information to the present cross section analyses over a wide range of photon energies.

While the results presented here are preliminary and our systematic uncertainties are currently at the $10 \%$ level, 
it is clear that the CLAS detector together with the photon tagger will have a large impact on the study of baryon
resonances in the near future.

Work performed with support from DOE Grant \# DE-FG05-95ER40901.

\section{REFERENCES}

1. R. P. Feynman, Photon-Hadron Interactions, Reading, Mass., W.A. Benjamin, 1972.

2. See references within: J. Ficenec et al, Jlab Experiment E94-103 (1994), www.jlab.org/

3. S.J. Brodsky, J.R. Hiller, Chueng-Ryong Ji, G.A. Miller, e-print: hep-ph/0105259 (200)/exp_prog/generated/apphallb.htm!

4. D. A. Jenkins and I. I. Strakovsky, Phys. Rev. C 52, 3499 (1995).

5. D.I. Sober et al, Nucl. Instrum. Methods A440,263 (2000),

6. E. Sinith et al., (to be published).

7. C. Cetina et al. Phys. Rev. Lett. 84 (2000) 5740-5743.

8. http://improv.unh.edu/Maurik/gsim_info.shtml

9. R.A. Amdt, I.I. Strakovsky, and R. L. Workman, Ptivate Communication 